\title{
POLAR DUALS OF CONVEX BODIES
}

\author{
MOSTAFA GHANDEHARI
}

(Communicated by William J. Davis)

\begin{abstract}
A generalization and the dual version of the following result due to Firey is given: The mixed area of a plane convex body and its polar dual is at least $\pi$. We give a sharp upper bound for the product of the dual crosssectional measure of any index and that of its polar dual. A general result for a convex body $K$ and a convex increasing real-valued function gives inequalities for sets of constant width and sets with equichordal points as special cases.
\end{abstract}

\section{INTRODUCTION}

Polar dual convex bodies are useful in geometry of numbers [19], Minkowski geometry $[12,13]$ and differential equations [14]. Chakerian [5] uses polar duals to discuss self-circumference of unit circles in a Minkowski plane.

The preliminary definitions and concepts used in this work are given in the next section. A generalization and dual version of the following result due to Firey [10] is given: The mixed area of a plane convex body and its polar dual is at least $\pi$. We give a sharp upper bound for the product of the dual crosssectional measure of any index and that of its polar dual. A general result for a convex body $K$ and a convex increasing real valued function gives inequalities for sets of constant width and sets with equichordal points as special cases.

\section{PRELIMINARIES}

By a convex body in $R^{n}, n \geq 2$, we mean a compact convex subset of $R^{n}$ with nonempty interior. All convex bodies are assumed to contain the origin in their interiors. For each direction $u \in S^{n-1}$ where $S^{n-1}$ is the unit sphere centered at the origin in $R^{n}$, we let $h(K, u)$ denote the support function of the convex body $K$ evaluated at $u$. Thus,

$$
h(K, u)=\sup \{u \cdot x: x \in K\},
$$

which may be interpreted as the distance from the origin to the supporting hyperplane of $K$ having outward-pointing normal $u$. The width of $K$ in

Received by the editors May 3, 1990; the contents of this paper were presented to the 839th meeting of the AMS, Atlanta, Georgia, January 1988.

1980 Mathematics Subject Classification (1985 Revision). Primary 52A40; Secondary 53A15.

Key words and phrases. Convex body, polar dual, mixed volume, dual mixed volume. 
direction $u$, denoted $W(K, u)$, is given by

$$
W(K, u)=h(K, u)+h(K,-u) .
$$

A convex body $K$ is said to have constant width $b$ if, and only if, $W(K, u)=$ $b$ for all $u \in S^{n-1}$. For a plane convex body $K$ we shall use the notation $h(K, \theta)=h(K, u)$, where $u=(\cos \theta, \sin \theta)$. In this case the width of $K$ in the direction $\theta$ can be written as

$$
W(K, \theta)=h(K, \theta)+h(K, \theta+\pi) .
$$

The polar dual (or polar reciprocal) of a convex body $K$, denoted by $K^{*}$, is another convex body having the origin as an interior point and is defined by

$$
K^{*}=\{y \mid x \cdot y \leq 1 \text { for all } x \in K\} .
$$

This definition depends upon the origin. If $K$ is the origin, then $K^{*}$ is the whole space. If $K$ is any other single point, then $K^{*}$ is a closed half space. The polar dual has the property that

$$
h\left(K^{*}, u\right)=1 / \rho(K, u) \text { and } \rho\left(K^{*}, u\right)=1 / h(K, u),
$$

where $\rho(K, u)$ and $\rho\left(K^{*}, u\right)$ denote radial functions of $K$ and $K^{*}$ respectively, defined by

$$
\rho(K, u)=\sup \left\{\lambda>0 \mid \lambda u \in K, u \in S^{n-1}\right\} .
$$

Let $B$ be the closed unit ball in $R^{n}$. The outer parallel set of $K$ at distance $\lambda>0$ is given by

$$
K_{\lambda}=K+\lambda B .
$$

The convex body $K_{\lambda}$ consists of all points in $R^{n}$ whose distance from $K$ is less than or equal to $\lambda$. It turns out that the volume $V\left(K_{\lambda}\right)$ is a polynomial in $\lambda$ whose coefficients are geometric invariants of $K$ :

$$
V(K+\lambda B)=\sum_{i=0}^{n}\left(\begin{array}{l}
n \\
i
\end{array}\right) W_{i}(K) \lambda^{i} .
$$

The functionals $W_{i}(K)$ do not have a standard name in English. In German $W_{i}(K)$ is the $i$ th Quermassintegral of $K$. It's rough translation is the $i$ th cross-sectional measure of $K$. Bonnesen and Fenchel [2] and Hadwiger [17] are standard references for the study of Quermassintegrals. The following is true:

$$
W_{0}(K)=V(K) ; \quad n W_{1}(K)=S(K) ; \quad W_{n}(K)=\omega_{n},
$$

where $V(K)$ and $S(K)$ are the volume and surface area of $K$ respectively and $\omega_{n}$ is the volume of the unit ball $B$ in $R^{n}$. It turns out that $W_{n-1}(K)$ has an interesting representation. The mean width of $K$, denoted by $\bar{W}(K)$ is given by

$$
\bar{W}(K)=\frac{1}{n \omega_{n}} \int_{S^{n-1}} W(K, u) d u
$$


where $d u$ is the area element of $S^{n-1}$. Then in fact

$$
W_{n-1}(K)=\frac{\omega_{n}}{2} \bar{W}(K)=\frac{1}{2 n} \int_{S^{n-1}} W(K, u) d u .
$$

By using (2) and (11), one obtains

$$
W_{n-1}(K)=\frac{1}{n} \int_{S^{n-1}} h(K, u) d u .
$$

The $W^{i}(K)$ are special cases of a set of functionals, depending on more than one convex body, introduced by Minkowski (in the three-dimensional case).

If $K_{1}, \ldots, K_{r}$ are convex bodies in $R^{n}$ and $\lambda_{1}, \ldots, \lambda_{r}$ range over the positive real numbers, then the volume of $\lambda_{1} K_{1}+\cdots+\lambda_{r} K_{r}$ is a homogeneous polynomial, of degree $n$, in $\lambda_{1}, \ldots, \lambda_{r}$. That is

$$
V\left(\lambda_{1} K_{1}+\cdots+\lambda_{r} K_{r}\right)=\sum a_{i_{1} \cdots i_{n}} \lambda_{i_{1}} \cdots \lambda_{i_{n}},
$$

where the coefficients $a_{i_{1} \ldots i_{n}}$ depend only on $K_{i_{1}}, \ldots, K_{i_{n}}$. We may assume that coefficients are chosen so as to be invariant under permutations of their subscripts. Then these coefficients are called mixed volumes and denoted by $a_{i_{1} \cdots i_{n}}=V\left(K_{i_{1}}, \ldots, K_{i_{n}}\right)$ to indicate their dependence on $K_{i_{1}}, \ldots, K_{i_{n}}$. We have, in other words,

$$
V\left(\lambda_{1} K_{1}+\cdots+\lambda_{r} K_{r}\right)=\sum V\left(K_{i_{1}}, \ldots, K_{i_{n}}\right) \lambda_{i_{1}} \cdots \lambda_{i_{n}},
$$

where $i_{1}, \ldots, i_{n}$ range independently over $1, \ldots, r$. Important properties of mixed volumes are discussed in Eggleston [8]. Chakerian [4] gives properties of mixed areas. It follows from (8) that

$$
W_{i}(K)=V(\underbrace{K, \ldots, K,}_{n-i} \underbrace{B, \ldots, B}_{i})
$$

which is sometimes used as a definition of $W_{i}(K)$.

The dual mixed volumes are defined in Lutwak [22], Burago and Zalgaller [3]

$$
\tilde{V}\left(K_{1}, \ldots, K_{n}\right)=\frac{1}{n} \int_{S^{n-1}} \rho\left(K_{1}, u\right) \cdots \rho\left(K_{n}, u\right) d u,
$$

where $d u$ signifies the area element on $S^{n-1}$. Let

$$
\tilde{V}_{i}\left(K_{1}, K_{2}\right)=\tilde{V} \underbrace{\left(K_{1}, \ldots, K_{1}\right)}_{n-i}, \underbrace{\left.K_{2}, \ldots, K_{2}\right)}_{i} .
$$

The dual cross-sectional measures are the special dual mixed volumes defined by

$$
\widetilde{W}_{i}(K)=\widetilde{V}_{i}(K, B),
$$

where $B$ is the unit ball in $R^{n}$. We shall use the following results of Lutwak

$$
\widetilde{W}_{i}(K) \leq V(K)^{(n-1) / n} \omega_{n}^{i / n},
$$


and

$$
\widetilde{V}^{n}\left(K_{1}, \ldots, K_{n}\right) \leq V\left(K_{1}\right) \cdots V\left(K_{n}\right) .
$$

After obtaining inequalities for mixed volumes and dual mixed volumes, we shall use the following definitions to prove geometric inequalities for sets of constant width and sets with equichordal, equiproduct and equireciprocal points. A point $P$ is an equichordal point of a convex region $K$ if and only if all the chords through $P$ have the same length. If the origin is an equichordal point with chord length 2 , then

$$
\rho(K, u)+\rho(K,-u)=2 .
$$

$P$ is an equiproduct point of a convex region $K$ if and only if each chord through $P$ intersects the boundary of $K$ at points $A$ and $B$ such that the product of $\overline{P A}$ and $\overline{P B}$ is constant. If the origin is an equiproduct point with constant 1 , then

$$
\rho(K, u) \cdot \rho(K,-u)=1 .
$$

$P$ is an equireciprocal point of a convex region $K$ if and only if each chord through $P$ intersects the boundary of $K$ in points $A$ and $B$ such that $1 / \overline{P A}+$ $1 / \overline{P B}$ is a constant. If the origin is an equireciprocal point with constant 2 , then

$$
1 / \rho(K, u)+1 / \rho(K,-u)=2 .
$$

Klee [23] has a discussion of sets with equichordal, equiproduct, or equireciprocal points.

$K$ is a set of constant relative width $b$ if, and only if,

$$
K+(-K)=b E
$$

where $E$ is the unit ball of a given Minkowski space ( $n$-dimensional Banach space).

\section{Results}

Theorem 1 below implies a generalization of the following result, due to Firey [10], as a special case: The mixed area of a plane convex body and its polar dual is at least $\pi$.

Theorem 1. Consider $n$ convex bodies $K, K_{1}, \ldots, K_{n-1}$ in $R^{n}$. Then the mixed volumes $V\left(K, K_{1}, \ldots, K_{n-1}\right), V\left(K^{*}, K_{1}, \ldots, K_{n-1}\right), V\left(B, K_{1}, \ldots, K_{n-1}\right)$ satisfy

$$
V\left(K, K_{1}, \ldots, K_{n-1}\right) V\left(K^{*}, K_{1}, \ldots, K_{n-1}\right) \geq V^{2}\left(B, K_{1}, \ldots, K_{n-1}\right) .
$$

Proof. By definition,

$$
V\left(K, K_{1}, \ldots, K_{n-1}\right)=\frac{1}{n} \int h(K, u) d S\left(K_{1}, \ldots, K_{n-1} u\right)
$$


and

$$
V\left(K^{*}, K_{1}, \ldots, K_{n-1}\right)=\frac{1}{n} \int h\left(K^{*}, u\right) d S\left(K_{1}, \ldots, K_{n-1}, u\right) .
$$

Multiply both sides of the above two equalities and use $h\left(K^{*}, u\right)=1 / \rho(K, u)$ and the Cauchy Schwarz inequality to obtain

$$
\begin{aligned}
n^{2} V & \left(K, K_{1}, \ldots, K_{n-1}\right) V\left(K^{*}, K_{1}, \ldots, K_{n-1}\right) \\
& =\left(\int h(K, u) d S\left(K_{1}, \ldots, K_{n-1}\right)\right)\left(\int \frac{1}{\rho(K, u)} d S\left(K_{1}, \ldots, K_{n-1}\right)\right) \\
& \geq\left(\int \sqrt{h(K, u)} \cdot \frac{1}{\sqrt{\rho(K, u)}} d S\left(K_{1}, \ldots, K_{n-1}\right)\right)^{2} \\
& \geq\left(\int d S\left(K_{1}, \ldots, K_{n-1}, u\right)\right)^{2}=n^{2} V^{2}\left(B, K_{1}, \ldots, K_{n-1}\right) .
\end{aligned}
$$

The last inequality follows since $h(K, u) \geq \rho(K, u)$.

Corollary 1.1. The mixed volume of $K$ and $K^{*}, V\left(K^{*}, K, \ldots, K\right)$ satisfies

$$
V\left(K^{*}, K, \ldots, K\right)^{n} \geq \omega_{n}^{2} V(K)^{n-2},
$$

where $\omega_{n}$ is the volume of an $n$-dimensional unit ball and $V(K)$ denotes the volume of $K$.

Proof. Let $K_{1}=K_{2}=\cdots=K_{n-1}=K$. Then (25) reduces to

$$
V(K) V\left(K^{*}, K, \ldots, K\right) \geq V^{2}(B, K, \ldots, K)=\left[\frac{1}{n} S(K)\right]^{2} .
$$

Use the general isoperimetric inequality,

$$
S^{n} \geq n^{n} \omega_{n} V^{n-1}
$$

to obtain (26).

The case $n=2$ gives Steinhardt's and Firey's result. The following result can be obtained from Theorem 1 as a special case.

Corollary 1.2. Let $K$ be a convex body and $K^{*}$ its polar dual then

$$
W_{n-1}(K) W_{n-1}\left(K^{*}\right) \geq \omega_{n}^{2} \text {. }
$$

Proof. Let $K_{1}=K_{2}=\cdots=K_{n-1}=B$ in Theorem 1. Use (15) and (25) to obtain (27).

The problem of finding the infimum of the product $W_{i}(K) W_{i}\left(K^{*}\right)$ for all convex bodies $K$, for each $i$, is not completely solved. See Bambah [1], Dvoretzky and Rogers [8], Firey [11], Guggenheimer [15, 16], Heil [18], Lutwak [22], and Steinhardt [24] for partial results. In Theorem 3 we use an inequality due to Blaschke-Santaló (see Theorem 2) concerning the product of volume of a convex body $K$ and its polar dual $K^{*}$ with respect to the Santaló point of $K$. The Santalo point of $K$ is often defined as the unique point in the interior of $K$ with respect to which the volume of the polar dual is a minimum. For a good discussion of the Blaschke-Santaló inequality and a further list of references, see Lutwak [21]. 
Theorem 2 (the Blaschke-Santaló inequality). Assume $K$ is a convex body in $R^{n}$ and $K^{*}$ is its polar dual with respect to the Santalo point of $K$. Then

$$
V(K) V\left(K^{*}\right) \leq \omega_{n}^{2},
$$

with equality if and only if $K$ is an ellipsoid.

Theorem 3. Let $K$ be a convex body in $R^{n}$. Assume $K^{*}$ is the polar dual of $K$ with respect to the Santalo point. Then the dual mixed volume of $K$ and $K^{*}, \widetilde{V}\left(K^{*}, K, \ldots, K\right)$, satisfies

$$
\widetilde{V}\left(K^{*}, K, \ldots, K\right)^{n} \leq \omega_{n}^{2} V(K)^{n-2} .
$$

Proof. By (20),

$$
\widetilde{V}^{n}\left(K^{*}, K, \ldots, K\right) \leq V\left(K^{*}\right) V(K)^{n-1} .
$$

Use Santaló's inequality,

$$
V(K) V\left(K^{*}\right) \leq \omega_{n}^{2},
$$

to obtain the desired inequality.

The case $n=2$ in Theorem 3 above gives a result similar to Firey's result. The dual mixed area of a plane convex body and its polar dual with respect to the Santaló point is at most $\pi$.

The following theorem concerning dual mixed volumes will generalize Santaló's inequality.

Theorem 4. Let $K_{1}$ and $K_{2}$ be two convex bodies. Assume $K_{1}^{*}$ and $K_{2}^{*}$ are the polar dual of $K_{1}$ and $K_{2}$ with respect to the Santalo points respectively. Then the dual mixed volumes $\widetilde{V}_{i}\left(K_{1}, K_{2}\right)$ and $\widetilde{V}_{i}\left(K_{1}^{*}, K_{2}^{*}\right)$ satisfy

$$
\tilde{V}_{i}\left(K_{1}, K_{2}\right) \widetilde{V}_{i}\left(K_{1}^{*}, K_{2}^{*}\right) \leq \omega_{n}^{2} .
$$

Proof. Lutwak [22] shows that

$$
\tilde{V}_{i}\left(K_{1}, K_{2}\right) \leq V\left(K_{1}\right)^{(n-i) / n} V\left(K_{2}\right)^{i / n}, \quad 0 \leq i<n .
$$

Replace $K_{i}$ by $K_{i}^{*},(i=1,2)$, to obtain

$$
\widetilde{V}_{i}\left(K_{1}^{*}, K_{2}^{*}\right) \leq V\left(K_{1}^{*}\right)^{(n-i) / n} V\left(K_{2}^{*}\right)^{i / n} .
$$

Multiply both sides of the above two inequalities and use Santaló's inequality to obtain the desired result.

If $K_{1}=K_{2}=K$ then (29) reduces to Santaló's inequality. If $K_{1}=K, K_{2}=$ $B$ then (29) reduces to the following corollary.

Corollary 4.1. Assume $K$ is a convex body in $R^{n}$. Assume $K^{*}$ is the polar dual of $K$ with respect to Santalo point. Then

$$
\widetilde{W}_{i}(K) \widetilde{W}_{i}\left(K^{*}\right) \leq \omega_{n}^{2} .
$$

Theorem 5 below is a general result which gives inequalities for sets of constant width and sets with equichordal point as special cases. See Chakerian and Groemer [6] for an excellent survey of sets of constant width. 
Theorem 5. For a convex body $K$ and convex increasing real valued function $\varphi$ define $g(K)$ by

$$
g(K)=\int_{S^{n-1}} \varphi(\rho(K, u)) d u .
$$

The functional $g$ satisfies

$$
g\left[\left(\frac{K_{1}+K_{2}}{2}\right)^{*}\right] \leq \frac{g\left(K_{1}^{*}\right)+g\left(K_{2}^{*}\right)}{2},
$$

and equality holds if and only if $K_{1}=K_{2}$.

Proof.

$$
\begin{aligned}
\frac{g\left(K_{1}^{*}\right)+g\left(K_{2}^{*}\right)}{2} & =\int_{S^{n-1}} \frac{\varphi\left(\rho\left(K_{1}^{*}, u\right)\right)+\varphi\left(\rho\left(K_{2}^{*}, u\right)\right)}{2} d u \\
& \geq \int_{S^{n-1}} \varphi\left[\frac{\rho\left(K_{1}^{*}, u\right)+\rho\left(K_{2}^{*}, u\right)}{2}\right] d u \\
& \geq \int_{S^{n-1}} \varphi\left[2\left(\rho\left(K_{1}^{*}, u\right)^{-1}+\rho\left(K_{2}^{*}, u\right)^{-1}\right)^{-1}\right] d u
\end{aligned}
$$

The first inequality uses the convexity of $\varphi$. The second inequality follows since $\varphi$ is increasing and the arithmetic mean is greater than or equal to the harmonic mean.

We now use (5) and the linearity property of the support function to obtain,

$$
\begin{aligned}
\int_{S^{n-1}} & \varphi\left[2\left(\rho\left(K_{1}^{*}, u\right)^{-1}+\rho\left(K_{2}^{*}, u\right)^{-1}\right)^{-1}\right] d u \\
& =\int_{S^{n-1}} \varphi\left[\left(\frac{h\left(K_{1}, u\right)+h\left(K_{2}, u\right)}{2}\right)^{-1}\right] d u \\
& =\int_{S^{n-1}} \varphi\left[\left(h\left(\frac{K_{1}+K_{2}}{2}, u\right)\right)^{-1}\right] d u \\
& =\int_{S^{n-1}} \varphi\left(\rho\left(\left(\frac{K_{1}+K_{2}}{2}\right)^{*}, u\right)\right) d u=g\left[\left(\frac{K_{1}+K_{2}}{2}\right)^{*}\right] .
\end{aligned}
$$

Thus, (31) follows. For equality to hold, it is necessary that $\rho\left(K_{1}^{*}, u\right)=$ $\rho\left(K_{2}^{*}, u\right)$ which implies $K_{1}=K_{2}$. For example, equality of the arithmetic and harmonic means of $\rho\left(K_{1}^{*}, u\right)$ and $\rho\left(K_{2}^{*}, u\right)$ implies $\rho\left(K_{1}^{*}, u\right)=\rho\left(K_{2}^{*}, u\right)$.

One can use (31) and continuity of $g$ to derive

$$
\frac{1}{P} \sum_{i=1}^{p} g\left(K_{i}^{*}\right) \geq g\left[\left(\frac{\sum K_{i}}{P}\right)^{*}\right]
$$

using the standard argument that leads to Jensen's inequality. More generally if $\left\{K_{t}: 0 \leq t \leq 1\right\}$ is a family of convex bodies and $K=\int_{0}^{1}\left(K_{t}\right) d t$ is the Minkowski-Riemann integral (see Dinghas [7]) then

$$
g\left(K^{*}\right) \leq \int_{0}^{1} g\left(K_{t}^{*}\right) d t .
$$


Corollary 5.1. The $n$-dimensional volume of the polar reciprocal of a set $K$ of constant relative width 2 satisfies

$$
V\left(K^{*}\right) \geq V\left(E^{*}\right) .
$$

Equality holds if and only if $K=E$, the unit ball in the given Minkowski space. Proof. Let $\varphi(t)=\frac{1}{n} t^{n}$. Then $\varphi$ is an increasing convex function. For any set $K, g(K)=V(K)$ where $g$ is defined as in Theorem 5. Hence using (31),

$$
\begin{aligned}
V\left(E^{*}\right) & =V\left(\left(\frac{K+(-K)}{2}\right)\right) \leq \frac{V\left(K^{*}\right)+V\left((-K)^{*}\right)}{2} \\
& =\frac{V\left(K^{*}\right)+V\left(K^{*}\right)}{2}=V\left(K^{*}\right) .
\end{aligned}
$$

By Theorem 5 equality holds if and only if $K=-K=E$.

Corollary 5.2. Let $K^{*}$ be the polar dual of a set $K$ of constant width 2 in $R^{n}$. Then

$$
W_{i}\left(K^{*}\right) \geq W_{i}(B), \quad i=0,1,2, \ldots, n-1,
$$

with equality if and only if $K$ is a unit ball.

Proof. By Corollary 5.1,

$$
V\left(K^{*}\right) \geq \omega_{n}
$$

with equality if and only if $K=B$. This is the case $i=0$ since $W_{0}\left(K^{*}\right)=$ $V\left(K^{*}\right)$. The well known classical inequality (see Hadwiger [17, p. 278]) shows that for any convex set $K$,

$$
W_{i}(K)^{n} \geq \omega_{n}^{i} V(K)^{n-i}
$$

replacing $K$ by $K^{*}$ in (37) and using (36) implies (35).

The following is an easy consequence of Corollary 5.2 for a set with an equireciprocal point.

Theorem 6. If $K$ is a convex set with an equireciprocal point corresponding to constant 2 then

$$
W_{i}(K)=W_{i}(B)=\omega_{n}, \quad i=0,1,2, \ldots, n-1,
$$

with equality if and only if $K$ is a unit ball centered at the origin.

Proof. (23) and (5) imply that $K^{*}$ is a set of constant width 2 . The fact that $\left(K^{*}\right)^{*}=K$ and Corollary 5.2 imply (38).

Theorem 7. If $K$ has an equichordal point with chord length 2 , then

$$
W_{n-1}\left(K^{*}\right) \geq \omega_{n},
$$

with equality if and only if $K$ is the unit ball centered at the origin.

Proof. The width of $K^{*}$ in direction $u$ satisfies

$$
W\left(K^{*}, u\right)=h\left(K^{*}, u\right)+h(K,-u) \geq \frac{4}{\rho(K, u)+\rho(K,-u)}=2,
$$


where we have used the inequality between arithmetic and harmonic means, (5), and (21). Then the mean width of $K^{*}$, denoted by $\bar{W}\left(K^{*}\right)$, satisfies

$$
\bar{W}\left(K^{*}\right)=\frac{1}{n \omega_{n}} \int_{S^{n-1}} W\left(K^{*}, u\right) d u \geq \frac{1}{n \omega_{n}} \int_{S^{n-1}} 2 d u \geq 2 .
$$

But

$$
W_{n-1}\left(K^{*}\right)=\frac{\omega_{n}}{2} \cdot \bar{W}\left(K^{*}\right) \geq \frac{\omega_{n}}{2} \cdot 2=\omega_{n},
$$

as we wanted to show. Equality holds if and only if $\rho(K, u)=\rho(K,-u)=1$, which implies $K$ is a unit ball centered at the origin.

Theorem 8. If $K$ has an equiproduct point with constant 1 , then

$$
W_{i}(K)=W_{i}(B)=\omega_{n}, \quad i=0,1,2, \ldots, n-1 .
$$

Proof. We first prove the case $i=0$; namely, $V(K) \geq \omega_{n}$. Together the inequality between arithmetic and geometric means and (22) imply

$$
\rho(K, u)+\rho(K,-u) \geq 2 \sqrt{\rho(K, u) \rho(K,-u)}=2 .
$$

Also,

$$
V(K)=\frac{1}{n} \int_{S^{n-1}}[\rho(K, u)]^{n} d u=\frac{1}{n} \int_{S^{n-1}} \frac{(\rho(K, u))^{n}+(\rho(K,-u))^{n}}{2} d u .
$$

Convexity of the function $x^{n}$ implies

$$
\frac{1}{n} \int_{S^{n-1}} \frac{(\rho(K, u))^{n}+(\rho(K,-u))^{n}}{2} d u \geq \frac{1}{n} \int_{S^{n-1}}\left[\frac{\rho(K, u)+\rho(K,-u)}{2}\right]^{n} d u .
$$

Equations (44), (45), and (46) imply the result for $i=0$, namely,

$$
V(K) \geq \frac{1}{n} \int_{S^{n-1}} d u=\frac{1}{n} n \omega_{n}=\omega_{n} .
$$

Equality holds if and only if $\rho(K, u)=\rho(K,-u)$. Using (21), equality holds if and only if $\rho(K, u)=\rho(K,-u)=1$ which gives a unit ball centered at the origin.

To prove (43) we use (37), noting that equality holds if and only if $K$ is the unit ball centered at the origin.

\section{ACKNOWLEDGMENT}

The author is thankful to the Naval Postgraduate School for support during this work.

\section{REFERENCES}

1. R. P. Bambah, Polar reciprocal convex bodies, Proc. Cambridge Philos. Soc. 51 (1955), 377-378.

2. T. Bonnesen and W. Fenchel, Theorie der konvexen Körper, reprint, Chelsea, New York, 1948. 
3. Yu D. Burago and V. A. Zalgaller, Geometric inequalities, Springer-Verlag, Heidelberg, 1988.

4. G. D. Chakerian, Geometric inequalities for plane convex bodies, Canad. Math. Bull. 22 (1979).

5. __ Mixed areas and the self-circumference of a plane convex body, Arch. Math. 34 (1980), 81-83.

6. G. D. Chakerian and H. Groemer, Convex bodies of constant width, convexity, and its applications (P. M. Gruber and J. M. Wills, eds.), Birkhäuser, 1983.

7. A. Dinghas, Minkowskische summen und integrale, Gauthier-Villars, Paris, 1961.

8. A. Dvoretzky and C. A. Rogers, Absolute and unconditional convergence in normed linear spaces, Proc. Nat. Acad. Sci. U.S.A. 36 (1950), 192-197.

9. H. G. Eggleston, Convexity, Cambridge Univ. Press, Cambridge, 1958.

10. W. J. Firey, The mixed area of a convex body and its polar reciprocal, Israel J. Math. 1 (1963), 201-202.

11. __ Support flats to convex bodies, Geom. Dedicata 2 (1973), 225-248.

12. H. Guggenheimer, The analytic geometry of the unsymmetric Minkowski plane, Lecture Notes, University of Minnesota, Minneapolis, 1967.

13. __ The analytic geometry of the Minkowski plane. I, A universal isoperimetric inequality, Abstract 642-697, Notices Amer. Math. Soc. 14 (1967), 121.

14. __ Hill equations with coexisting periodic solutions, J. Differential Equations 5 (1969), 159-166.

15. __, Polar reciprocal convex bodies, Israel J. Math. 14 (1973), 309-316.

16. __ Corrections to Polar reciprocal convex bodies, Israel J. Math. 29 (1978), 312.

17. H. Hadwiger, Vorlesungen über Inhalt, Oberfläche und Isoperimetrie, Springer-Verlag, Berlin, 1957.

18. E. Heil, Ungleichungen für die Quermassintegrale Polarer Körper, Manuscripta Math. 19 (1976), 143-149.

19. C. G. Lekkerkerker, Geometry of numbers, Wolters-Noordhoff, Groningen, 1969.

20. E. Lutwak, On cross-sectional measures of polar reciprocal convex bodies, Geom. Dedicata 5 (1976), 79-80.

21. __ Blaschke-Santaló inequality, discrete geometry and convexity, Ann. New York Acad. Sci. 440 (1985), 106-112.

22. __ Intersection bodies and dual mixed volumes, Adv. in Math. 71 (1988), 231-261.

23. V. Klee, Shapes of the future-some unsolved problems in geometry, Part I, Two dimensions, Film available MAA, 1971.

24. F. Steinhardt, On distance functions and on polar series of convex bodies, Ph.D. Thesis, Columbia Univ., 1951.

Department of Mathematics, Naval Postgraduate School, Monterey, California 93943 